\title{
Renewable energy sustainability in terms of systems analysis
}

\author{
Vladimir Kovalev*, Olga Arkhipova, and Alexander Paramzin \\ Yugra State University, Chekhova str., 16, 628011 Khanty-Mansiysk, Russia
}

\begin{abstract}
The article deals with the application of the principles of systems analysis in the study of sets of isolated power supply systems within the tasks of sustainable development. For such aggregates, in terms of systems analysis, the concept of a regionally isolated electrotechnical complex is introduced. The analyzed electrotechnical complex is proved to be a complex production and technological system. With regard to a complex production and technological system - a regionally isolated electrotechnical complex, the authors provide a meaningful interpretation of the following principles of the theory of systems analysis: consistency, connectivity, hierarchy, emergence, optimality, and equifinality.
\end{abstract}

\section{Introduction}

One of the goals of sustainable development, proposed by the UN in 2015 , is "Goal 7: Ensure access to affordable, reliable, sustainable and modern energy for all" (SDG7) [1].

This gave rise to social, economic, and environmental subtasks [2-4]. The power industry, as one of the responses to this challenge, has intensified its activity in renewable energy sources [5]. The renewable energy source (RES) supporting schemes built in a number of countries [6] have led to a systemic reduction in the cost of electricity produced by RES. Only in 2010-2015, the electricity generated by solar panels decreased by $50 \%$ [7], the forecasted decrease by 2025 is another $57 \%$ of the achieved. Wind power is being improved through systemic optimization, both of the wind generator itself, and through the energy-saving upgrade of the entire wind farm infrastructure. The forecast of a decrease in the cost of electricity produced with the use of renewable energy sources allows us to expect an expansion of its volume and geography, including in places with no pronounced natural and climatic conditions favorable for the use of RES.

An inextricable and interdependent triad of "production-consumption-storage" of energy resources, in contrast to the classical construction of energy supply according to a dual "production-consumption" scheme is becoming a key characteristic of RES energy supply. Considering the stochastic nature of the energy produced by renewable energy sources requires "transformation of the development vector" [8] of the entire electric power generation, transportation, and consumption complex. This requires changes in design, construction, and management principles for all components interacting in the specified energy conversion process. With regard to the energy infrastructure of the Russian

\footnotetext{
${ }^{*}$ Corresponding author: vz_kovalev@mail.ru
} 
Federation, the implementation of SDG7 urgently requires moving to a higher level of supply of electrical and other types of energy to "remote and isolated territories" [9, pp. 5455]. At the same time, the Energy Strategy of the Russian Federation [9] sets a trend for a steady decrease in the production cost of " $1 \mathrm{kWh}$ of electricity in the territories of decentralized power supply" [9, p. 55].

Using renewable energy sources in the production of electric power becomes a tool that optimizes the task of power supply to settlements located in the territories of decentralized power supply. The development of renewable energy proceeds in several directions. The primary is the use of wind and solar energy. Such types of renewable energy sources as geothermal, tidal, and wave are actively developed. Although the total share of renewable energy consumed in the world is less than $2 \%$, there has been an intensive growth in the installed capacities of both wind and solar energy, about $600 \%$, in the last decade. The expected growth rates until 2040 are $6.3-8.3 \%$ per year. In the long term, it is the "further development of RES" [10] that will determine the main changes in the global structure of electric power generation and consumption.

\section{Materials and Methods}

Let a regionally isolated electrotechnical complex (RIETC) be a set of isolated power supply systems (IPSS) united by some complex-inherent features [11]. The main specific features of RIETC, partially described in [12] and supplemented in this work, include the following:

- each IPSS of the considered aggregate is an electrotechnical generation and consumption complex (ETGC), which at the same time converts external energies of various types (chemical, thermal, solar, hydraulic, ..., hereinafter referred to as "external energy influence" (EEI)) into electrical energy consumed by a separate IPSSs;

- $\quad$ all IPSSs included in the analyzed RIETC interact with each other only through "mutual weak relations and weak interactions" [11], hereinafter referred to as "external non-energy influences" (ENI);

- all IPSSs included in the RIETC operate on the basis of some integral ultimate goal, conditioned by the requirements of a suprasystem or supersystem that subordinates RIETC [12] and interacts with RIETC through energy and non-energy connections;

- non-energy influences inherent in the analyzed RIETC, affect the quality of functioning of the entire RIETC (including energy parameters) in the operating range of external influences.

External non-energy interactions between the IPSS of the considered RIETC will be further understand to mean a characteristic set of interactions and interdependencies by means of information technologies in administrative, legal, social, and other similar spheres. The allocation of ENI into a separate group of indicators is due, on the one hand, to their intangible nature, and on the other hand, to an indirect influence on the basic properties of RIETC as an object of generation and consumption of electrical energy. Here we can indicate the ENI-dependent indicators: the quality of electrical energy, the composition and parameters of the generating elements of RIETC, the energy efficiency of the IPSS included in the RIETC, the volume of consumed resources, and reliability indicators. We should separately note, for the considered set of IPSS, the dependence of such indicators on ENI as the cost and environmental friendliness of electric power production. Thus, based on the above features of the RIETC, we should consider the external non-energy influences along with energy influences already at the design stage of the RIETC and its IPSSs.

The design and engineering surveys and technological procedures during the operation of RIETC require an optimization approach. In this case, complications arise in 
mathematical modeling, associated with the need to consider the interactions and interdependencies carried out by different physical forces. A solution is often sought through the use of a finite element network [13]. The "chain-field" problems can be provided as an example of a fairly effective application of this method, where a combination of computing packages is used, for example, ANSYS and Matlab. At the same time, these approaches do not directly consider the revealed weak relations and weak interactions. This requires either the use of decomposition-based approaches with a natural decrease in the hierarchy of the resulting model [14], or the development of a specialized approach.

\section{Results and Discussion}

The introduced definition of RIETC and its characteristic features imply as follows: the ultimate operation goal of RIETC, as a set of IPSSs, is the reliable provision of consumers being part of IPSS with electrical power of the required quality. The actual production and transmission of electrical power to the consumer, as well as the management of these processes, is carried out through the interaction and mutual influence of physically dissimilar components of IPSSs included in the RIETC. As previously noted, the quality of functioning of the IPSSs included in the ROETC also significantly depends on the totality of external non-energy interactions inherent in the considered RIETC. Accordingly, we can state as follows:

Statement 1. RIETC is a complex consisting of functionally independent and physically dissimilar components that interact and interfere with each other. The functions of all components of RIETC and the RIETC itself as a whole are aimed at achieving the supersystem set goal or goals. The interaction and interdependence of the components of the RIETC proceeds through a system of external energy and non-energy interactions [12].

The above statement largely reflects the definition of a complex system given in GOST 22.2.04-2012 [10], and correlates with a number of definitions adopted in the world and domestic literature [11-14], including the concept of a complex technical system. The corresponding outline structural energy flowchart of RIETC CTS in terms of systems analysis is shown in Figure 1.

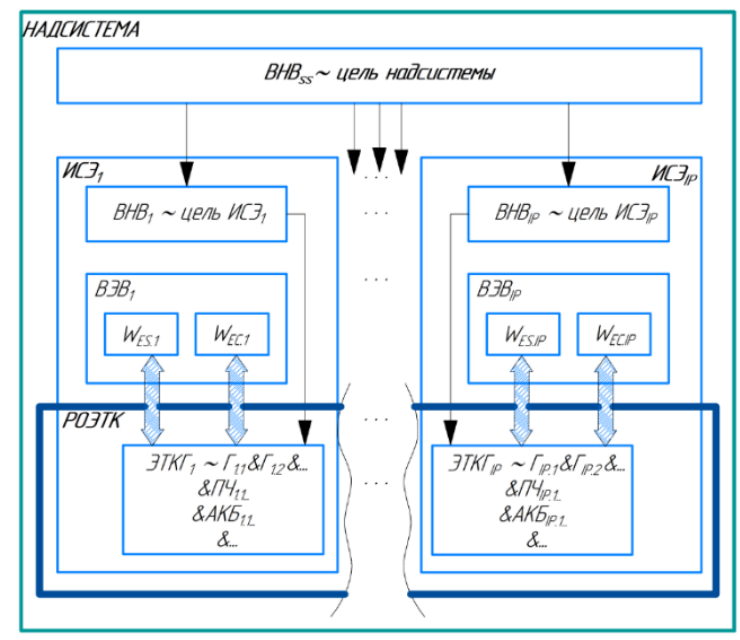

Fig. 1. The outline structural energy flowchart of RIETC CTS in terms of systems analysis (IPSS isolated power supply system, EEI - external energy influence, ENI - external non-energy influence, $\mathrm{W}$ - types of energies) 
Were: Надсистема-Supersystem; BHB- ENI; ВЭВ- EЕI; Цельнадсистемы - Supersystem goal; ИСЭ- IPSS; ЦельИСЭ - IPSS goal; РОЭТК- RIETC; ЭТКГ - ETGC (electrotechnical graphitization complex); ПЧ - FC (frequency converter); АКБ - АВ (accumulator battery); $\Gamma$ - G (graphitization)

Statement 1 leads to the need to use systems analysis (MSA) in the analysis and synthesis of ROETK CTS [12]. Optimization problems for ROETK CTS, using MSA, lead to the need for a weakly formalized choice of optimality criteria in a multidimensional space. Additionally, it becomes necessary to synthesize an algorithm for cutting off the set of optimal search alternatives. Note that the MSA approaches are quite well developed, including in the field of large power systems $[14,15]$. However, the above structural and functional features of ROETK CTS, as a specific complex technical system, lead to the need to adapt the conceptual apparatus of MSA. Primarily - "the MSA basic principles: the principle of consistency, the principle of connectivity, the principle of hierarchy, the principle of optimality, the principle of emergence, the principle of equifinality, and the concept of an integral target indicator of the ROETK CTS operation (ITI)" [12].

Further formulations are integrative, and consider both the key characteristics and features of RIETC CTS substantiated above, and the theoretical provisions of a number of literary sources [12, 16-25].

The integral target indicator of the RIETC CTS operation is a certain norm, consisting of indicators that ensure the required quality and reliability of power supply, and including the EEI and ENI inherent in the considered RIETC CTS. The optimal, in a certain sense, the ITI value is achieved by solving a multiparameter optimization problem corresponding to Statement 1.

The principle of consistency. Here the RIETC CST is understood to mean a complex object consisting of a set of interrelated and mutually influencing components of different physical nature. Interconnection and interaction is carried out through EEI and ENI. This principle requires the study of the RIETC CTS itself, subject to the interaction and mutual influence with a larger system (supersystem), which interacts with the RIETC complex technical system through weak relations and weak interactions.

The principle of connectivity. The analysis of any component of the RIETC CTS reveals and considers both internal relations between the components included in the RIETC CTS and interactions and mutual influences with the external environment (considering the various external influences). In this case, the RIETC CTS is primarily considered as an element of the super-system.

The principle of hierarchy. Here the RIETC CTS is understood to mean a hierarchical set of physically heterogeneous subsystems. Sub-systemization is possible both by the features of the physical boundaries of the components entering the system and by the type of energy converted in the power channel, which is reflected by the energy structural diagram [26]. The assignment of a component, or a group of components to a certain hierarchical level, is determined by the functioning purpose of this level, which is set by a component or group of components of a higher level. In this case, the interaction of all components, regardless of their physical nature, is determined by conservation laws.

The principle of optimality. Here, the criterion of ITI compliance is applied to the functioning of the RIETC CTS throughout the entire life cycle, within the framework of permissible changes in external influences. The mathematical model of RIETC CTS is built as a multi-parameter system with a common goal, in accordance with Statement 1.

The principle of emergence. Both EEI and ENI, inherent in the RIETC CTS in accordance with Statement 1, lead to the emergence of new integrative properties, previously absent in any of the components formally included in the RIETC CTS. For example, a conductor and a magnetic field. Only with mutual displacement, that is, when combined into a system, a new property appears - electromotive force. With regard to the design and operation of the RIETC CTS, this circumstance leads to the emergence of 
significant local optima, which, as a rule, do not coincide with the global optimum determined by the design of the ITI.

Note that the complex multilevel hierarchical structure of RIETC only aggravates the problem of finding the global optimum and requires developing problem-oriented optimization methods.

The principle of equifinality. Regardless of the adopted initial conditions, the adopted trajectory of motion, external energy influences and external non-energy influences, the RIETC CTS is able to provide optimal requirements for the indicators of its functioning (energy, social, environmental) set by the integral properties of the system and the design of the integral target indicator. A practical consequence of this principle is the multivariate design of the ITI and the development algorithm of the RIETC CTS.

\section{Conclusions}

1. The main specific features of RIETC have been defined.

2. RIETC has been proved to be a complex production and technological system (RIETC CTS).

3. With regard to RIETC CTS, the basic principles of system analysis have been adapted: the principle of consistency, the principle of connectivity, the principle of hierarchy, the principle of optimality, the principle of emergence, and the principle of equifinality.

\section{Acknowledgements}

The research was performed with financial support of the Russian Foundation for Basic Research and the Government of Khanty-Mansiysk Autonomous District - Yugra in the framework of the research project No.18-47-860017.

\section{References}

1. Technical report by the Bureau of the United Nations Statistical Commission (UNSC). Date Views 17.09.2020, https://sustainabledevelopment.un.org/

2. N. Spittler, G. Gladkykh, B. Davidsdottir, A. Diemer, Energies, 8, 1584 (2019)

3. J. Baleta, H. Mikulčić, N. Duić, J.J. Klemeš, K. Urbaniec, Journal of Cleaner Production, 215, 1424 (2019)

4. F. Fuso Nerini, C. Spataru, G. Anandarajah, J. Tomei, L.S. To, M. Black, Y. Mulugetta, I. Bisaga, P. Parikh, A. Borrion, V. Castán Broto, B. Milligan, Nature Energy, 1,10 (2019)

5. S.O. Oyedepo, T. Uwoghiren, P.O. Babalola, O. Kilanko, R.O. Leramo, A.K. Aworinde, T. Adekeye, S.C. Nwanya, J.A. Oyebanji, O.A. Abidakun Open Engineering, 1, 72 (2019)

6. L. Lehoux, H. Duck, R. Akhmadeev, T. Morozova, O. Bykanova, Journal of Security and Sustainability, 2, 457 (2019)

7. International Renewable Energy Agency, IRENA 2016; The Power to Change: Solar and Wind Cost Reduction Potential to 2025 (2016)

8. S.A. Nekrasov, I.D. Grachev. Problems of forecasting, 1, 99 (2020) 
9. Energy Strategy of the Russian Federation until 2035, Ministry of Energy website, https://minenergo.gov.ru/

10. V.V. Elistratov, Ecological Bulletin of Russia, 11, 30 (2017)

11. O.V. Arkhipova, V.Z. Kovalev, R.N. Khamitov, Bulletin of Tomsk Polytechnic University. Engineering of georesources,1, 173 (2019)

12. O.V. Arkhipova, Omsk Scientific Bulletin,3(171), 42 (2020)

13. E. G. Andreeva, Journal of Physics: Conference Series,1050, 012003 (2018)

14. E. G. Andreeva, A. S Gritsay, Journal of Physics: Conference Series, 052001 (2019)

15. GOST 22.2.04-2012 Safety in emergency situations. Man-made accidents and disasters. Metrological support for monitoring the state of complex technical systems. Basic provisions and rules. Introduction (2019)

16. V.Ia. Tsvetkov, Educational resources and technologies. Complex technical systems, 3(20), 86 (2017)

17. A.Iu. Loskutov, A.S. Mikhailov, Izhevsk: Institute of Computer Research, 620 (2007)

18. M.D. Mesarovic, Yasuhiko Takahara, General Systems Theory: Mathematical Foundations Academic Press, New York San Francisco London 1975 A Subsidiary of Harcourt Brace Jovanovieh, Publishers, 316

19. V.A. Barishpolets, Modeling, decomposition, and optimization of complex dynamic processes. Application of the main provisions of the general theory of systems in program-target planning and management of the creation of complex technical and man-machine systems, 1 (33),43 (2018)

20. Voropai N.I., Theory of systems for electric power industry: Textbook, 273 (2000)

21. B.V. Papkov, A.L. Kulikov, The theory of systems and system analysis for electric power industry: textbook and workshop for undergraduate and graduate programs, 2nd ed. (2019), https://urait.ru/bcode/434717

22. Orazbaev B.B., Kurmangazieva L.T., KodanovaSh.K., Theory and methods of systems analysis: textbook (2017)

23. V.N. Volkova, V. N. Kozlov, System modeling. Approaches and methods: textbook(2013)

24. E. A. Godovnikov, Journal of Physics: Conference Series, 1260, 052007 (2019)

25. V. Z. Kovalev, Journal of Physics: Conference Series, 1260, 052014 (2019)

26. Kovalev V.Z., Malgin G.V., Arkhipova O.V., Mathematical modeling of electrotechnical complexes of oil and gas production within the energy saving tasks (2008) 